CONTRIBUCIÓN DEL $3^{\text {ER }}$ CONGRESO LATINOAMERICANO DE MACROINVERTEBRADOS DE AGUA DULCE: BIODIVERSIDAD Y ECOLOGÍA FUNCIONAL EN EL NEOTRÓPICO

\title{
BIOMASA DE ODONATOS EN UN HUMEDAL ARTIFICIAL SUBURBANO, CAJICÁ (CUNDINAMARCA-COLOMBIA)
}

\author{
BIOMASS OF ODONATA IN A SUBURBAN ARTIFICIAL WETLAND, CAJICÁ \\ (CUNDINAMARCA-COLOMBIA)
}

José A. Cuellar-Cardozo y María I. Castro-Rebolledo

\begin{abstract}
RESUMEN
Los humedales de la Sabana de Bogotá presentan modificaciones ecológicas causadas por el impacto de las actividades antropogénicas. La productividad secundaria, particularmente de macroinvertebrados como los odonatos, es uno de los aspectos más importantes que permite definir con precisión el grado de conservación de los humedales. Este trabajo tuvo como objetivo caracterizar el ensamble de odonatos a través de la identificación taxonómica, la densidad y la cuantificación de la biomasa y evaluar una relación alométrica entre el crecimiento de la amplitud cefálica y el peso seco de náyades de Rhionaeschna marchali (Rambur, 1842) (Aeshnidae), presentes en un humedal suburbano de la Universidad Militar Nueva Granada (Cajicá, Cundinamarca). En total se realizaron siete muestreos semanales en el periodo comprendido entre agosto y octubre del 2014. Se obtuvieron datos con relación a la densidad, la biomasa y un modelo de regresión lineal entre el peso seco y la amplitud cefálica, para el caso de larvas de Rhionaeschna marchali $\left(\mathrm{R}^{2}=0,61\right)$, las demás regresiones no fueron significativas. La especie Mesamphiagrion laterale (Selys, 1876) (Coenagrionidae) mostró la mayor densidad de individuos adultos (0,14 ind $\mathrm{m}^{-2}$ ) y Rhionaeschna marchali $\left(200\right.$ ind $\mathrm{m}^{-2}$ ) la mayor densidad de inmaduros.
\end{abstract}

PALABRAS CLAVE: peso seco, ecuaciones alométricas, modelo de regresión, amplitud cefálica, longitud corporal

\begin{abstract}
The wetlands from Sabana of Bogota have ecological alterations caused by the impact of anthropogenic activities. Second productivity particularly in macroinvertebrates like the odonates, is the most important aspect that can precisely define the conservation degree of wetlands. This study aimed to characterize the Odonata assembly through taxonomic identification, density and biomass quantification, and to evaluate an allometric relation between the growth of the cephalic amplitude and the dry weight of Rhionaeschna marchali (larvae), present in a suburban wetland from Nueva Granada Military University (Cajicá, Cundinamarca). In total seven weekly samplings were made in the period between August and October 2014. Data were obtained in relation to the density, biomass and a linear regression model between the dry mass and cephalic amplitude for Rhionaeschna marchali (Rambur, 1842) (Aeshnidae) naiads $\left(\mathrm{R}^{2}=0.61\right.$ ), the others regressions were not significant. Mesamphiagrion laterale (Selys, 1876) (Coenagrionidae) showed the highest density of adult individuals $\left(0.14 \mathrm{ind}^{-2}\right.$ ) and Rhionaeschna marchali (200 ind $\mathrm{m}^{-2}$ ) had the highest density of larvae.
\end{abstract}

KEY WORDS: dry mass, secondary productivity, regression model, cephalic amplitude, body length 
Los humedales de la Sabana de Bogotá poseen una alta productividad primaria (Sánchez y Amat, 2005), la cual no alcanza a ser consumida ni mineralizada y en consecuencia se acumula en los ambientes acuáticos (Márquez, 2003). Esto ocasiona, la acumulación de la materia orgánica y la eutrofización. En los últimos años, la conservación de los humedales se ha visto afectada por el acelerado crecimiento industrial y urbano de la región (Rivera-Usme et al., 2013), lo que ha afectado de gran manera la presencia de estos sistemas acuáticos.

Los humedales suburbanos se caracterizan por ser ecosistemas que presentan grandes extensiones de terreno cubiertas por vegetación acuática y alta influencia antropogénica (Kutcher y Bried, 2014). Dentro de este tipo de humedales viven gran cantidad de macroinvertebrados, siendo el estudio de su productividad secundaria uno de los aspectos que define con mayor grado de precisión el estado de conservación de los humedales (Rivera-Usme et al., 2013). Allí, los odonatos son componentes básicos de la red trófica, ya que son depredadores en todos sus estados de desarrollo y gracias a esto son parte de la dieta de otros animales (Clauznitzer et al., 2009). Por lo que son organismos importantes en la transferencia de energía en los ecosistemas acuáticos (Crowley et al., 1987) y su biomasa se convierte en una variable que permite estimar, tanto la producción primaria, como la secundaria de un ecosistema (Meyer, 1989). Sin embargo, la biomasa de los odonatos ha sido poco estudiada si la comparamos con datos predictivos de otros invertebrados y los estudios con relación a la biomasa de imagos son casi nulos (Smock, 1980). Debido a la falta de información referente a la producción secundaria de los odonatos en sistemas neotropicales; el objetivo de este estudio fue determinar la densidad y la biomasa de estos organismos, en un humedal andino del campus Cajicá de la Universidad Militar Nueva Granada (UMNG) en Cundinamarca, Colombia.

El estudio se realizó en el campus Cajicá de la UMNG 4 56' 33,39” N 74 0' 46,24” O, 2550 m de altitud, ubicado en la ronda del río Bogotá (Figura 1). El humedal tuvo un origen antrópico, provocado por la formacion de diques que separaron parte del plano inundable del río Bogotá; en este se observó la presencia de macrófitas acuáticas tales como: Bidens laevis, Hydrocotyle ranunculoides, Azolla filiculoides, Schoenoplectus californicus, Dryopteris wallichiana, Juncus effusus, Lemna gibba, Phytolacca bogotensis, Holcus lanatus, Polygonum hydropiperoides, Cenchrus clandestinus. Limnobium laevigatum fue la especie dominante.

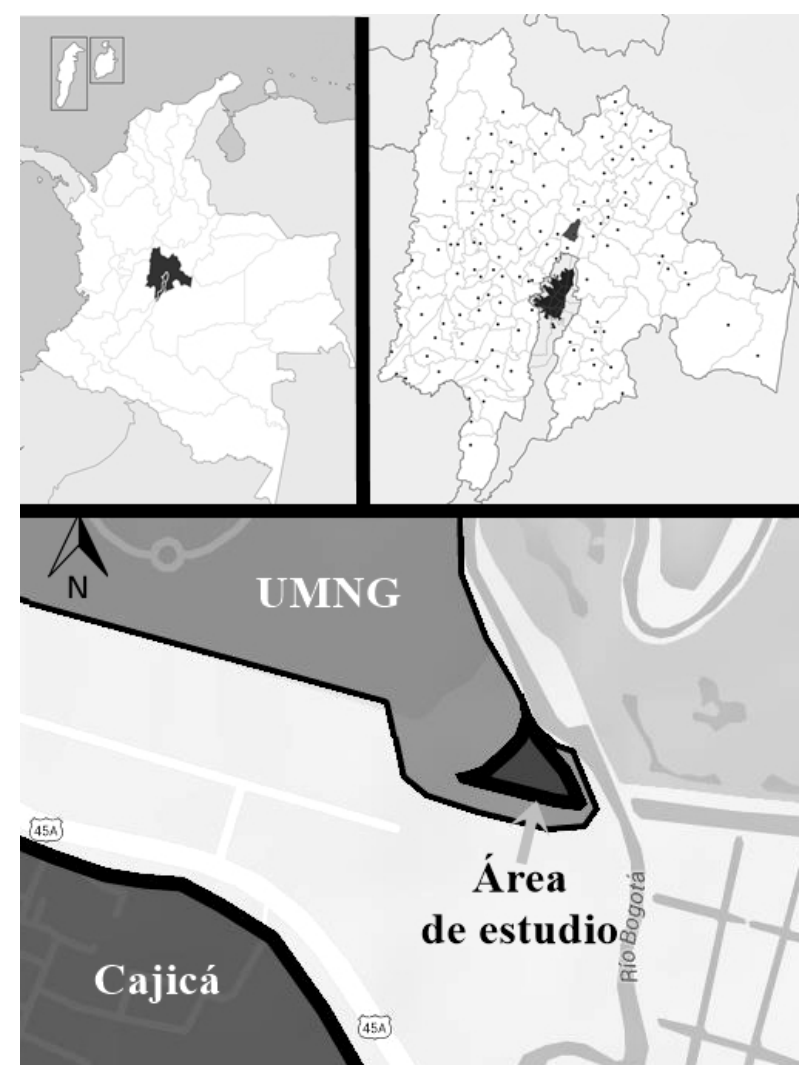

Figura 1. Localización del humedal del campus Cajicá Universidad Militar Nueva Granada.

En el primer muestreo, se realizó la toma de datos fisicoquímicos del humedal. A una profundidad de 0,5 $\mathrm{m}$ y por medio de una sonda multiparamétrica marca $H A N N A^{\circledR}$, se registraron los datos de $\mathrm{pH}$, temperatura $\left({ }^{\circ} \mathrm{C}\right)$, conductividad $\left(\mu \mathrm{S} \mathrm{cm} \mathrm{cm}^{-1}\right)$ y oxígeno disuelto $\left(\mathrm{mg} \mathrm{L}^{-1}\right)$.

La recolección del material biológico se efectuó entre los meses de agosto a octubre de 2014. Estos meses presentaron valores de precipitación entre 19 a $48 \mathrm{~mm}$ $\mathrm{mes}^{-1}$. En total se realizaron siete muestreos, los cuales se llevaron a cabo semanalmente en el horario de 09:00 $\mathrm{h}$ a las 16:00 h.

La recolecta de imagos se realizó por medio de redes entomológicas, en un área correspondiente a $400 \mathrm{~m}^{2}$, ubicada en la ribera del humedal. Para la captura de larvas, se utilizó una red de recolección de macroinvertebrados asociados a macrófitas acuáticas con un área de $400 \mathrm{~cm}^{2}$ y un ojo de malla de $63 \mu \mathrm{m}$. En cada muestreo se realizaron cinco réplicas teniendo en cuenta que se llevaron a cabo en una zona litoral uniforme. 
La preservación de los individuos recolectados se hizo por medio del montaje en seco para el caso de imagos y en viales con alcohol etílico al 70 \% para las larvas. Los individuos recolectados se registraron y almacenaron en la colección entomológica de la UMNG-Cajicá (Universidad Militar Nueva Granada). La identificación taxonómica se llevó a cabo hasta el nivel taxonómico más detallado posible. Para los imagos, la identificación se hizo con base en la información y claves taxonómicas de von Ellenrieder (2003); Garrison et al. (2006); von Ellenrieder y Garrison (2008); Garrison et al. (2010); Pérez y Palacino (2011) y Bota-Sierra y Echeverri (2013). Para las larvas se recurrió a la información y las claves taxonómicas de Geijskes (1941); De Marmels (2007); Merritt et al. (2008) y Ramírez (2010).

La densidad de los odonatos presentes en el humedal se estimó por cada $\mathrm{m}^{2}$ de área de captura. La biomasa se estimó en las especies que presentaron una cantidad significativa de individuos ( $>40)$ : Rhionaeschna marchali (Rambur, 1842) (40 larvas) y Mesamphiagrion laterale (Selys, 1876) (55 imagos), por medio de regresiones lineales bivariadas. A estos especímenes, se les midió la longitud total del cuerpo (LC), la cual se definió como la longitud desde el clípeo hasta el último segmento abdominal, descartando la pirámide anal en las larvas y la genitalia en los imagos y la amplitud máxima de la amplitud cefálica (AC) de acuerdo a lo planteado por Smock (1980) y Burgherr y Meyer (1997), sin discriminar entre machos y hembras. Las mediciones morfométricas se realizaron con un estereoscopio y con reglilla micrométrica marca Olympus ${ }^{\circledR}$ con una precisión de 0,12 mm. Siguiendo el método de Burgherr y Meyer (1997), los organismos se llevaron a un horno para su secado $\left(48 \mathrm{~h}\right.$ a $70^{\circ} \mathrm{C}$ ) y se obtuvo su peso seco con una precisión de 0,01 $\mu$ g. Los datos se ajustaron a una regresión lineal mediante el uso del programa SPSS $20^{\circledR}$.

Debido a la baja abundancia de larvas e imagos de los otros grupos de odonatos recolectados ( $R$. marchali (adultos), M. laterale (náyades) e Ischnura cruzi (De Marmels, 1987) (adultos y náyades)), los valores de biomasa se establecieron por medio de la masa individual promedio (Smock, 1980).

Los principales parámetros fisicoquímicos registrados en el humedal se presentan en la tabla 1.

En total, se recolectaron 110 individuos de odonatos pertenecientes a las especies $R$. marchali, $M$. laterale e I. cruzi (Figura 2). Las densidades estimadas (ind $\mathrm{m}^{-2}$ ) para cada uno de los estadios de las especies recolectadas se presentan en la tabla 2 , en donde se observa la diferencia entre los datos registrados y el estadio en que se muestrearon los organismos. La densidad más alta en la etapa adulta fue para la especie M. laterale $\left(0,14\right.$ ind $\left.\mathrm{m}^{-2}\right)$ y para estadio larval fue $R$. marchali (200 ind $\mathrm{m}^{-2}$ ).

Tabla 1. Valores de las principales características fisicoquímicas registradas en el humedal del campus Cajicá -Universidad Militar Nueva Granada.

\begin{tabular}{lr}
\hline Parámetro & Valor \\
\hline $\mathrm{pH}$ & 6,65 \\
Temperatura del agua $\left({ }^{\circ} \mathrm{C}\right)$ & 14,90 \\
Conductividad $\left(\mu \mathrm{Scm}^{-1}\right)$ & 477 \\
Oxígeno disuelto $\left(\mathrm{mg} \mathrm{l}^{-1}\right)$ & 5,35 \\
\hline
\end{tabular}

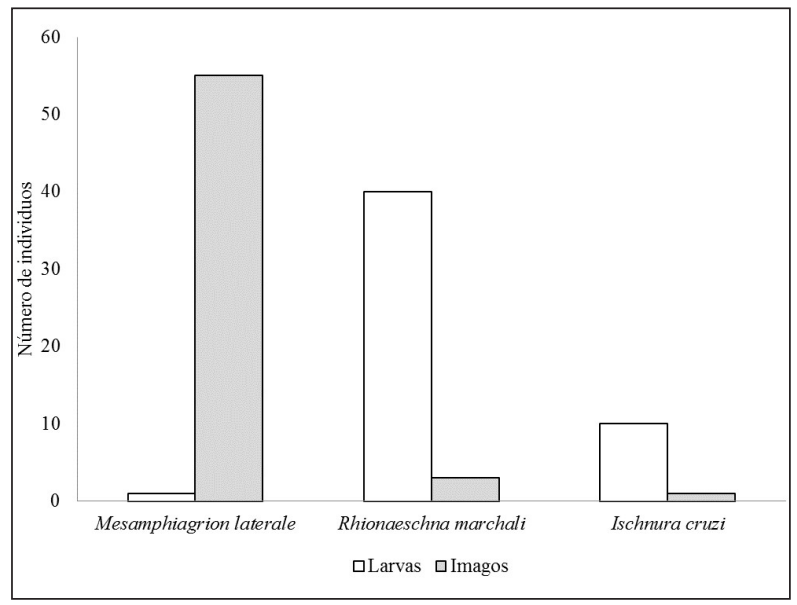

Figura 2. Números de individuos de odonatos recolectados en el humedal de la UMNG, entre agosto a octubre de 2014.

Los ejemplares medidos de $R$. marchali presentaron una AC entre 4,2 y $9,38 \mathrm{~mm}$, un LC entre 18,76 y 46,34 mm y un peso seco entre 8,76 y 173,1 mg. La regresión lineal simple entre $\mathrm{AC}$ y el peso seco de los organismos de $R$. marchali mostró un valor predictivo del $61 \%\left(\mathrm{R}^{2}\right.$ $=0,61 ; \mathrm{p}<0,05$ ) (Figura 3) (Tabla 3). Por otra parte, la regresión lineal entre LC y peso seco mostró un valor predictivo del $12 \%\left(\mathrm{R}^{2}=0,12 ; \mathrm{p}<0,05\right)$, por lo que se descarta una relación entre longitud corporal y peso seco del individuo.

De igual forma, los modelos de regresión lineal para la AC y LC de los imagos de M. laterale mostraron 
coeficientes de determinación muy bajos $\left(R^{2}=0,12\right.$ y $\mathrm{R}^{2}=0,06$, respectivamente) lo que permite establecer una alta afinidad alométrica entre estas mediciones y el peso seco (Tabla 3). Además, se observó que la relación entre $\mathrm{AC}$ y el peso seco presentó una pendiente casi nula $(0,07)$, mientras que la relación entre $\mathrm{LC}$ y el peso seco presentó una pendiente negativa $(-0,52)$, lo que indica una leve relación (inversa) entre el peso seco y el tamaño del organismo.

Tabla 2. Valores de densidad estimada (ind $\mathrm{m}^{-2}$ ) y biomasa (mg) de odonatos observados en el humedal de la UMNG, entre agosto a octubre de 2014 .

\begin{tabular}{lrrrr}
\hline & \multicolumn{2}{c}{ Densidad (ind m $\left.^{-2}\right)$} & \multicolumn{2}{c}{ Biomasa (mg) } \\
\hline Especie & Larvas & Imagos & Larvas & Imagos \\
\hline $\begin{array}{l}\text { Mesamphiagrion } \\
\text { laterale }\end{array}$ & 5 & 0,1375 & 35,780 & 447,830 \\
$\begin{array}{l}\text { Rhionaeschna } \\
\text { marchali }\end{array}$ & 200 & 0,0075 & 791,300 & 798,789 \\
\begin{tabular}{l} 
Ischnura cruzi \\
\hline
\end{tabular} & 50 & 0,0025 & 73,746 & 58,980 \\
\hline
\end{tabular}

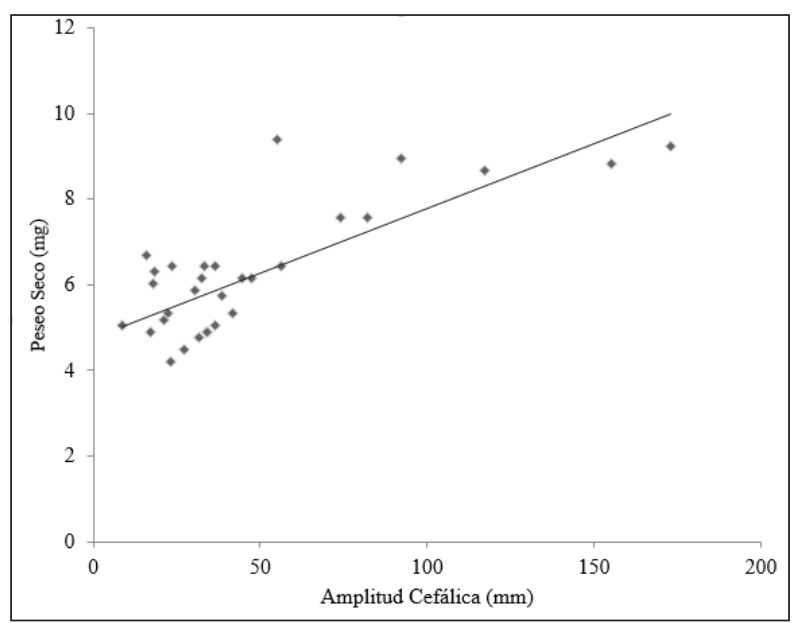

Figura 3. Regresión lineal entre la amplitud cefálica y el peso seco de las larvas de Rhionaeschna marchali.

Los valores de la biomasa estimada para las náyades de cada especie (Tabla 2), mostraron un mayor valor para $R$. marchali, con 791,3 $\mathrm{mg} \mathrm{m}^{-2}$, mientras que I. cruzi presentó un valor de $73,75 \mathrm{mg} \mathrm{m}^{-2}$. La única larva observada de M. laterale registró un valor de $35,78 \mathrm{mg} \mathrm{m}^{-2}$.
En el caso de los imagos (Tabla 2), R. marchali obtuvo el mayor valor de biomasa, aunque exhibió una menor densidad de individuos, en contraste con $M$. laterale que obtuvo la mayor densidad en los adultos, presentó valores bajos de biomasa. Los especímenes de I. cruzi mostraron el menor valor de biomasa.

En el presente trabajo se evidenció una alta densidad de los imagos de $M$. laterale y una baja abundancia de sus náyades, lo que podría indicar que el ciclo de vida de estos organismos pueden estar en proceso de reproducción sincronizado, por lo que la mayor parte de las náyades emergieron juntas (Cordero-Rivera, 2002). Según Garré (2014), esto es consecuencia del multivoltinismo de las especies de Coenagrionidae presentes en zonas tropicales. Asimismo, la estructura vegetal del humedal, dominada por L. laevigatum, incide en la presencia de imagos (Corbet, 2004; Córdoba-Aguilar, 2008), puesto que este tipo de plantas acuáticas proveen un buen refugio ante el clima y los depredadores (Bota-Sierra y Echeverri, 2013). Cabe resaltar que la alta densidad de $M$. laterale también altera las poblaciones de otros coenagriónidos adultos, debido a la competencia interespecifica (De Block y Stocks, 2005); posiblemente, este factor, junto con la presión selectiva por parte de los depredadores y la dispersión de individuos (Córdoba-Aguilar, 1993), inciden en la baja densidad de los imagos de I. cruzi encontrados en el presente estudio. A diferencia de $M$. laterale, la baja abundancia de adultos de $R$. marchali podría ser producto del poco éxito de captura.

Comparado con el estudio de Pallares y Monroy (2012) (Tabla 4), nuestro trabajo evidenció una poca abundancia de náyades de $M$. laterale que se relaciona con escasez de espejos de agua presentes en el humedal de la UMNG. El caso contrario ocurre con las náyades de $R$. marchali, que al igual que con los adultos de $M$. laterale, fueron favorecidas por la cobertura vegetal del humedal (von Ellenrieder, 2003; Corbet, 2004). También se evidenció lo planteado por Schaffner y Anholt (1998), donde la presencia de larvas de la familia Aeshnidae, como $R$. marchali, es inverso a la abundancia de náyades de I. cruzi.

En cuanto a la relación entre la Amplitud Cefálica y el peso seco para las náyades de $R$. marchali, se halló un valor predictivo del $61 \%$, que de acuerdo a Benke et al. (1999), permite el uso del modelo en 
humedales andinos. En el caso de los adultos de $M$. laterale, las medidas utilizadas para el modelo (AC y LC), no fueron adecuadas para elaborar una relación lineal con el suficiente valor predictivo, sin importar el sexo de los individuos, por lo que se recomienda usar otras medidas, como la morfología alar, que pueden estar más relacionadas con el peso seco del individuo. De acuerdo a Sabo et al. (2002) y Serrano-Meneses et al. (2007), la biomasa de imagos se ve afectada por variables ambientales y genéticas, y no por la densidad de los organismos en el ecosistema; mientras que en las náyades, la densidad sí está relacionada con este factor, lo que permite comprender parte del balance energético en el ecosistema, debido a la fuerte interrelación de las mismas con el sistema acuático (Folsom y Collins, 1982).

Tabla 3. Resumen de las regresiones lineales bivariadas, realizadas en náyades de $R$. marchali e imagos de M. laterale. (p <0.05)

\begin{tabular}{lrrrrrr}
\hline Especie & $\begin{array}{r}\text { Número de } \\
\text { especímenes }\end{array}$ & Medidas & Ecuación & b & m & $\mathbf{R}^{2}$ \\
\hline \multirow{2}{*}{ R. marchali } & \multirow{2}{*}{40} & Amplitud cefálica & $\mathrm{Y}=4,7687+0,0301 \mathrm{X}$ & 4,77 & 0,03 & 0,61 \\
& & Longitud corporal & $\mathrm{Y}=30,111+0,0746 \mathrm{X}$ & 30,11 & 0,07 & 0,12 \\
\multirow{2}{*}{ M. laterale } & \multirow{2}{*}{55} & Amplitud cefálica & $\mathrm{Y}=4,2798+0,0752 \mathrm{X}$ & 4,28 & 0,07 & 0,12 \\
& & Longitud corporal & $\mathrm{Y}=47,091-0,5242 \mathrm{X}$ & 47,09 & $-0,52$ & 0,06 \\
\hline
\end{tabular}

Tabla 4. Abundancia de náyades de odonatos de presente estudio y el estudio de Pallares y Monroy (2012) en el humedal La Vaca y Santa María del Lago.

\begin{tabular}{lrrr}
\hline & $\begin{array}{c}\text { Presente } \\
\text { estudio }\end{array}$ & Pallares y Monroy (2012) \\
\hline Especie & $\begin{array}{r}\text { Humedal } \\
\text { UMNG }\end{array}$ & La Vaca & $\begin{array}{r}\text { Santa María } \\
\text { del Lago }\end{array}$ \\
\hline $\begin{array}{l}\text { Mesamphiagrion } \\
\text { laterale }\end{array}$ & 1 & 25 & 5 \\
$\begin{array}{l}\text { Rhionaeschna } \\
\text { marchali }\end{array}$ & 40 & 33 & 61 \\
\begin{tabular}{l} 
Ischnura cruzi \\
\hline
\end{tabular} & 10 & 30 & 39 \\
\hline
\end{tabular}

En síntesis, se halló una relación lineal entre el crecimiento de la amplitud cefálica y el peso seco de las larvas de $R$. marchali. Sin embargo, en imagos de $M$. laterale fue difícil establecer esta relación debido, posiblemente, a su dependencia con otras variables, ambientales y genéticas. Se hace necesario intensificar el muestreo en otras épocas del año para hacer la comparación a lo largo de todo el ciclo de vida de los individuos.

\section{AGRADECIMIENTOS}

Agradecimientos a Silvia Morales por su ayuda en la fase de laboratorio y a Gabriel Pinilla, Cornelio Bota y Jhon Charles Donato por la revisión del manuscrito. De igual manera, agradecemos al laboratorio de entomología de la UMNG, por su colaboración y por el préstamo de materiales durante la ejecución del proyecto, y a la Vicerrectoría de Campus Cajicá de la UMNG por permitir la realización de los muestreos.

\section{BIBLIOGRAFÍA}

Benke, A., Huryn, A., Smock, L. y Wallace, J. 1999. Lengthmass relationships for freshwater macroinvertebrates in North America with particular reference to the southeastern United States. Journal North American Benthology Society 18(3): 308-343.

Bota-Sierra, C. y Echeverri, M. 2013. Taxonomic revision of Mesamphiagrion Kennedy, 1920 from Colombia (Odonata: Coenagrionidae), with the description of four new species. Zootaxa 3718(5): 401-440.

Burgherr, P. y Meyer, E. 1997. Regression analysis of liner body dimensions vs dry mass in stream macroinvertebrates. Archiv für Hydrobiologie 39(1): 101-112. 
Clauznitzer, V., Kalkman, V.J., Ram, M., Collen, B., Baillie, J., Bedjanic, M., Darwall, W., Dijkstra, K-B., Dowf, R., Hawking, J., Karube, H., Malikova, E., Paulson, D., Schütte, K., Suhling, F., Villanueva, R., von Ellenrieder, N. y Wilson, K. 2009. Odonata enter the biodiversity crisis debate: the first global assessment of an insect group. Biological Conservation 142: 1864-1869.

Corbet, P.S. 2004. Dragonflies Behaviour and Ecology of Odonata (Revised Edition). Harley Books, Colchester.

Cordero-Rivera, A. 2002. Influencia de la selección sexual sobre el comportamiento reproductor de los Odonatos. En: Soler, M., Editor. Evolución: la base de la biología. Proyecto Sur de Ediciones, S.L., Granada.

Córdoba-Aguilar, A. 1993. Population structure in Ischnura denticollis (Burmeister) (Zygoptera: Coenagrionidae). Odonatologica 22(4): 455-464.

Córdoba-Aguilar, A. 2008. Dragonflies and Damselflies, model organisms for ecological and evolutionary research. Oxford University Press, Oxford.

Crowley, P.H., Nisbet. P., Gurney, R. y Lawton, J. 1987. Population regulation in animals with complex lifehistories: formulation and analysis of a damselfly model. Advances in Ecological Research 17: 1-59.

De Block, M. y Stoks, R. 2005. Fitness effects from egg to reproduction: bridging the life history transition. Ecology 86(1): 185-197.

De Marmels, J. 1987. Ischnura (Anomalagrion) cruzi sp. n., eine neue Kleinlibelle aus Kolombien (Odonata: Coenagrionidae). Mitteilungen Entomologischen Gesellschaft Basel 37(1): 1-6.

De Marmels, J. 2007. Thirteen new Zygoptera larvae from Venezuela (Calopterygidae, Polythoridae, Pseudostigmatidae, Platystictidae, Protoneuridae, Coenagrionidae). Odonatologica 36(1): 27-51.

Folsom, T. y Collins, N. 1982. Food availability in nature for the larval dragonfly Anax junius (Odonata: Aeshnidae). Freshwater Invertebrate Biology 1(3): 33-40.

Garrison, R., von Ellenrieder, N. y Louton, J. 2006. Dragonfly Genera of the New World. The Johns Hopkins University Press, Baltimore.

Garrison, R., von Ellenrieder, N. y Louton, J. 2010. Damselfly Genera of the New World, an Illustrated and Annotated Key to the Zygoptera. The Johns Hopkins University Press, Baltimore.
Garré, A. 2014. Biología de las larvas de Cyanallagma interruptum Selys (1876) (Odonata: Coenagrionidae) Tesis de Doctorado, Universidad Nacional de La Plata, La Plata, Argentina.

Geijskes, D.C. 1941. Notes on Odonata of Surinam. II. Six mostly new zygopterous nymphs from the coastland waters. Annals of the Entomological Society of America 34: 719-734.

Kutcher, T.E. y Bried, J.T. 2014. Adult Odonata conservatism as an indicator of freshwater wetland condition. Ecological Indicators 38: 31-39.

Márquez, G. 2003. Bienes y servicios ecológicos de los humedales. En: Acueducto de Bogotá y Conservación Internacional - Colombia., Editores. Los humedales de Bogotá y la Sabana. Tomo II. Acueducto de Bogotá, Bogotá.

Merritt, R.W., Cummins, K.W. y Berg, M.B. 2008. An introduction to the aquatic insects of North America. Fourth edition. Kendall/Hunt Publishing Company, Dubuque.

Meyer, E. 1989. The relationship between body length parameters and dry mass in running water invertebrates. Archiv für Hydrobiologie 117(2): 191-203.

Pallares, M.I.M. y Monroy, G.H.G. 2012. Distribución espacial y temporal de náyades de odonatos en los humedales la Vaca y Santa María del Lago, Bogotá, Colombia. Acta Biológica Colombiana 17: 281-294.

Pérez, L.A. y Palacino, F. 2011. Updated checklist of the Odonata known from Colombia. Odonatologica 40(3): 203-225.

Rambur, M.P. 1842. Histoire Naturelle des Insectes Névroptères. Librairie encyclopédique de Roret, Paris.

Ramírez, A. 2010. Odonata. En: Springer, M., Ramírez, A. y Hanson, P., Editores. Macroinvertebrados de agua dulce de Costa Rica I. Revista de Biología Tropical 58(Suppl 4): 97-136.

Rivera-Usme, J.J., Pinilla-Agudelo, G.A. y Rangel, J.O. 2013. Ensamblaje de macroinvertebrados acuáticos y su relación con las variables físicas y químicas en el humedal de Jaboque - Colombia. Caldasia 35(2): 389-408.

Sabo, J., Bastow, J. y Power, M. 2002. Length-mass relationships for adult aquatic and terrestrial invertebrates in a California watershed. Journal North American Benthology Society 21(2): 336-343. 
Sánchez, D. y Amat, G. 2005. Diversidad de la fauna de artrópodos terrestres en el humedal Jaboque, Bogotá Colombia. Caldasia 27(2): 311-329.

Schaffner, A.K. y Anholt, B.R. 1998. Influence of predator presence and prey density on behavior and growth of damselfly larvae (Ischnura elegans) (Odonata: Zygoptera). Journal of Insect Behavior 11(6): 793-809.

Selys, E. 1876. Synopsis des Agrionines, 5me légion: Agrion (suite). Le genre Agrion. Bulletin de l'Académie royale de Belgique 41: 247-322.

Serrano-Meneses, M., Azpilicueta-Amorin, M., Szekely, T. y Córdoba-Aguilar, A. 2007. The development of sexual differences in body size in Odonata in relation to mating systems. European Journal of Entomology 104: 453-458.
Smock, L. 1980. Relationships between body size and biomass of aquatic insects. Freshwater Biology 10: 375-383.

von Ellenrieder, N. 2003. A synopsis of the Neotropical species of 'Aeshna' Fabricius: The Genus Rhionaeschna Förster (Odonata: Aeshnidae). Tijdschrift voor Entomologie 146: 67-207.

von Ellenrieder, N. y Garrison, R.W. 2008. Oreiallagma gen. nov. with a redefinition of Cyanallagma Kennedy 1920 and description of $M$. dunklei sp. nov. and $M$. ecuatoriale sp. nov. form Ecuador (Odonata: Coenagrionidae). Zootaxa 1805: 1-51.

Fecha de recepción: 15/09/2016

Fecha de aceptación: 17/11/2016

Para citar este artículo: Cuellar-Cardozo, J.A. y Castro-Rebolledo, M.I. 2016. Biomasa de odonatos en un humedal artificial suburbano, Cajicá (Cundinamarca-Colombia).

Revista Intropica Vol. 11: 97 - 103 